\title{
Mechanical Strength of Porous Catalyst Carriers
}

\author{
1.C. van den Born \\ Koninklijke/Shell-Laboratorium, Amsterdam (Shell Research B.V.) \\ P.O. Box 3003, 1003 AA Amsterdam, the Netherlands
}

\section{Abstract}

In order to extend our fundamental knowledge on the deformation and fracture of porous, amorphous particles under compressive and shear forces, we have developed a computer model within the framework of percolation theory. This 'computational physics' approach, which has been chosen especially in view of the complex nature of the fracture mechanism, would not have been feasible without parallel-computing facilities.

Experimental data from Side Crushing Strength (SCS) tests on porous catalyst carriers show a wide variance in elasticity and breaking strength. We should like to find the physical parameters that determine these properties.

The computer model is based on a two-dimensional network of Hooke-type springs with an eigenlength and a load limit. We have calculated the configuration of the spring network under a macroscopic strain. Individual springs that are stretched beyond a critical length break irreversibly. The implementation of the model on a Transputer network will be discussed.

The stress-strain relations of an SCS test and our model show a similar behaviour. The force goes up linearly with the relative compression unless a breakage occurs. The system breaks into two main parts after one or two avalanches of breakages.

\section{Introduction}

The mechanical strength of amorphous material has recently been receiving increased international attention. In contradiction to the description of linearresponse behaviour (elasticity, conductivity) it is believed that the mechanical strength of heterogeneous material cannot be modelled by an effective medium approximation. Since a propagating crack interacts strongly with its surroundings there is an intrinsic spread in the mechanical strength of non-crystalline material; the strength seems to have a statistical nature. Samples of identically prepared porous amorphous material might have mechanical strengths that vary by an order of magnitude.

Although in practice a deformation experiment on a rigid body seems to be of a three-dimensional nature, we shall only discuss two-dimensional deformation experiments in this paper. We plan to look at three-dimensional deformation experiments in the future. In two dimensions the mechanical linear-response behaviour 
of a structure is fully determined by two independent deformations. Other deformations can be seen as a combination of these two. We choose to characterise the structure with the Young modulus $Y$ and the shear modulus $\mu$. The Young modulus is the proportion between the pressure $p$, i.e. the perpendicular-force density, and the strain, the relative shortening in the direction of the pressure.

$$
p=\frac{F_{\text {perp }}}{\mathrm{A}}=y \frac{\Delta \mathrm{u}}{\mathrm{u}}
$$

The shear modulus is the proportion between the parallel-force density $g$ and the angle of deformation $\theta$.

$$
g=\frac{F_{\text {parallel }}}{\mathrm{A}}=\mu \theta
$$

If we increase the force density, non-linear effects can be observed. This might be a form of plasticity, i.e. a decrease in the derivative of the stress with respect to the strain, or a failure, i.e. a discontinuity in the stress as a function of the strain. We want to understand the connection between the microscopic properties of the amorphous structure and the maximal force it is able to resist.

We are especially interested in understanding the behaviour of catalyst carriers as observed in the so-called Side Crushing Strength (SCS) test. In the SCS test a single particle is slowly deformed by a plunger which pushes with a constant velocity, while the force needed is recorded. The catalyst carriers are made of porous amorphous silicon oxide. The production mechanism of these catalyst carriers causes a wide range in characteristics. The catalyst carriers look more or less round and have an average diameter of 2-3 $\mathrm{mm}$. There is a large variance in porosity. Even after eliminating these effects, the SCS test results seem to have a statistical nature. After selecting catalyst carriers within a small range of size and weight, we performed about $50 \mathrm{SCS}$ tests. The results are presented in Fig. 1. Beyond the spread in elasticity there is a variance in the ultimate strength as well. The cloud of points indicates that some kind of correlation between these observables exists. Similar spreads in results have been obtained in deformation experiments on composites and polymer systems [1].

We aim for a better understanding of the fracture mechanism in (porous) amorphous material. Due to the production mechanism there will always occur a spread in properties within an ensemble of catalyst carriers. A theoretical model would give us the opportunity to concentrate on the importance of a specific parameter. Later on these results could be embedded in a mathematical model to be used for interpreting and guiding deformation experiments on real amorphous material.

There are several ways to describe the breaking phenomena in amorphous structures theoretically. In the first place one can divide the global structure into finite elements by giving each finite element specific properties such as stiffness and a yield criterion, the fracture mechanism can be observed using finite elements methods. Another approach looks at the problem from a microscopic point of view 


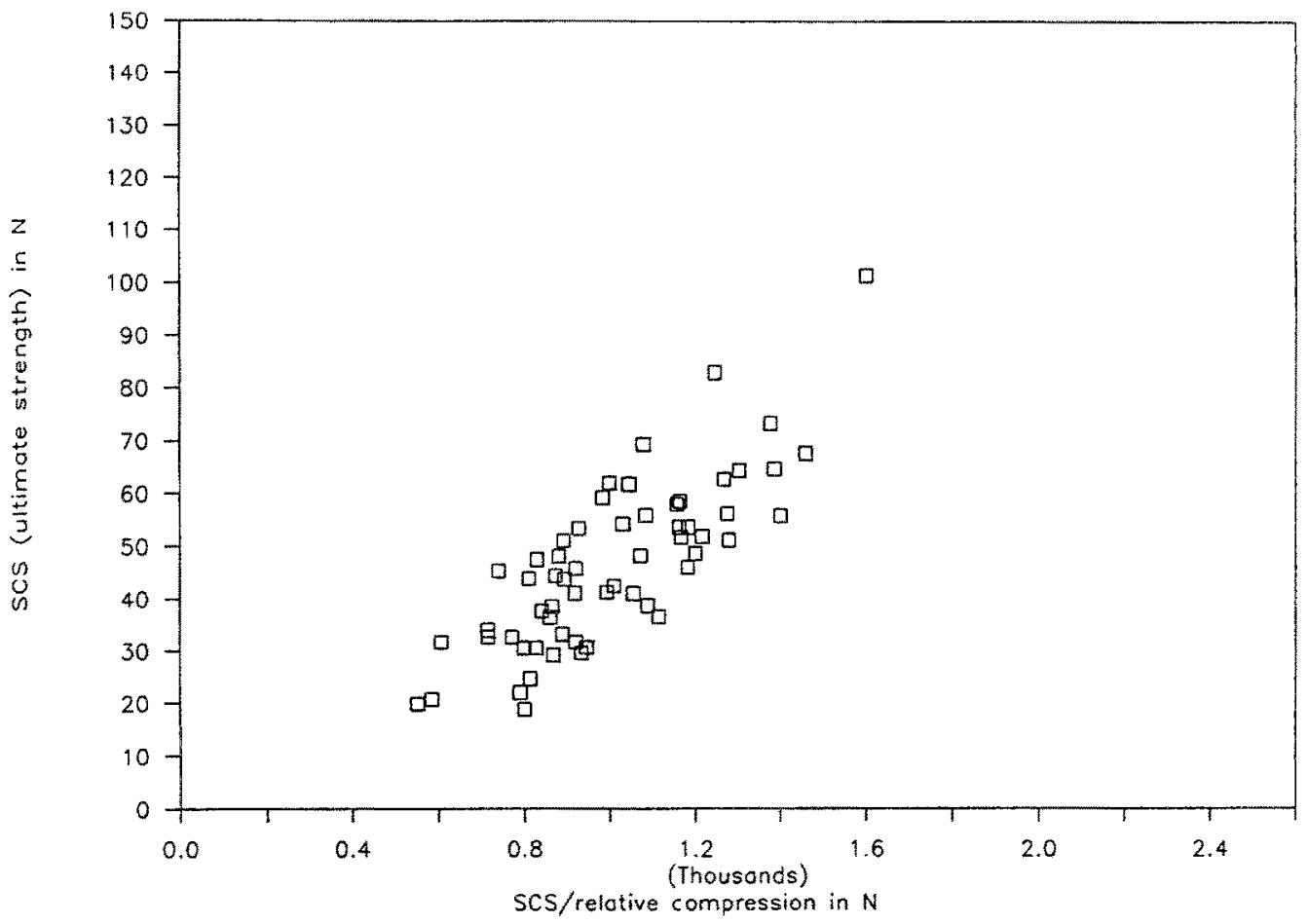

(Fig.1) Results of the SCS tests on porous amorphous catalyst carriers (silicon oxide) within a small range of size and weight.

and makes use of quantum chemistry. Here, the amorphous structure is built up from atoms. An increment of strain implies that the distances between neighbouring atoms will change according to their interatomic potential function. If the distance becomes too large the force of attraction gets too weak and the bond will break. A third way to describe the fracture mechanism stands midway between the preceding ones. The amorphous structure is modelled by randomly cutting a number of bonds in an artificial regular grid. This approach within the framework of percolation theory is most suitable for our problem. The statistical nature of the strength properties makes it inevitable that a lot of data have to be calculated. To gather these data cheaply and quickly, parallel-computing facilities can/must be used. Because of its regular grid and simplicity the percolation model lends itself admirably to simulating a deformation experiment.

In the next chapter we shall give some more information on percolation theory. After that the model and its parallel implementation are discussed. This paper is completed with a summary of some preliminary results.

\section{Percolation theory}

In order to obtain more fundamental knowledge on the deformation and fracture of porous, amorphous particles under compressive and shear forces, we have devel- 
oped a computer model. The output of the model will be interpreted with percolation theory [2]. Percolation theory describes the global behaviour of disordered material. It has been successfully used to describe for instance the electrical properties of random multiphase material and the diffusion of a liquid into a porous structure. The application to the mechanical behaviour of these materials is relatively new. The main aspect of the theory is that the calculations are made on an artificial network. The effective properties, rescaled on the number of nearest neighbours, are only dependent on the dimensionality of the structure. Finite-size scaling theories have been developed to interpret numerical results of calculations on finite lattices.

It is out of the scope of this paper to give an extensive introduction on percolation theory. Therefore we recommend the excellent book of Stauffer [2] . In order to get some feeling for percolation theory, we should like to treat the electrical breakdown model, which is developed to describe the electrical failure of disordered material. There are some authors who extend the results of the electrical breakdown model to describe the mechanical failure as well. At the end of this chapter we shall give arguments against the latter approach.

If one puts a mix of conducting and isolating balls into a box, one can simulate the electrical behaviour of porous amorphous material. The percentage of isolating balls is a measure of the porosity. Since it is easier to make numerical simulations on regular lattices, a better approach is to start with a square network of resistors. The porosity is introduced by giving a certain percentage of the resistors an infinite resistance. Distributing the 'porosity' in a random way results in an amorphous structure. As mentioned before, there is a difference between the results of the two models due to the fact that the number of neighbours of a ball in a random packing is different from the number of neighbours in a square grid. But after rescaling, the results are identical. We must, however, be careful since finite-size effects may influence the results considerably. The calculation to be done is to solve the system of linear equations given by Kirchoff's rules. This determines the total resistance of the network defined as the ratio between the voltage and the current. This percolation model is able to simulate the electrical linear-response behaviour of an amorphous material. Effective medium approximation techniques are able to describe these properties as well. The main advantage of the percolation model is that we can extend it to the non-linear response behaviour. The electrical breakdown of the structure can be simulated by introducing a critical current for every resistor in the network. If the current through a resistor exceeds the critical current, the resistor will break down. In the simulation, this breakdown is effected by giving the resistor an infinitely large resistance.

We can now observe the following behaviour. There exists a linear relationship between the current and the voltage up to a certain critical voltage. Then there will be a drop in the total current through the network due to the breakdown of a single resistor, followed by a redistribution of the current. This redistribution may lead to a failure elsewhere in the network. This may be the start of an avalanche of failures leading to a global breakdown.

Extrapolating the results from the electrical breakdown model to a mechanical breakdown model is very dangerous. In the first place there is only a change in the distribution of the current if failure occurs. In real mechanical deformation experiments, there are besides changes in the stress distribution local deformations of the material itself. Apart from this, it is nearly impossible to extrapolate the results 
from the electrical breakdown model not only to structures under tensile load but also to structures under compressive or shear load. The spring model introduced in the next chapter is able to simulate these deformation experiments directly.

\section{Elastic spring models}

\section{Conventional approaches}

Sahimi and Goddard [3] have introduced a class of models for cohesive failure in disordered solids, based on networks of Hooke-type springs with a load limit. They placed such a network under a macroscopic strain. After calculation of the network configuration, springs that are stretched beyond a critical length break irreversibly. They find, that the macroscopic behaviour, from ductile to brittle breakage, depends on the form of the distribution of the spring constant and the critical length. They determined the effective shear modulus of porous systems and found a difference between the behaviour of an unbroken system, i.e. a system with an initial porosity that is randomly distributed and the behaviour of a broken system, i.e. a system with an initial lower porosity where some cracks are already present.

However, we want to simulate the attrition of a porous material under a compressive rather than a tensile load. Therefore we need to make some modifications to the previous model. The most important one is the introduction of an eigenlength of every spring. Thus, if we now compress the system we need a force. Again the configuration of the network is calculated and springs which are stretched beyond the critical length are broken.

By introducing a non-vanishing eigenlength of a spring, we stepped outside the class of problems that can be solved using linear algebra routines. Prior to the introduction of an eigenlength the hamiltonian, i.e. the energy function, of the spring system, could be written as

$$
H=\frac{C}{2} \sum_{\text {springs }}\left[\left(x_{\text {begin }}-x_{\text {end }}\right)^{2}+\left(y_{\text {begin }}-y_{\text {end }}\right)^{2}\right]
$$

The equilibrium state is the minimal energy configuration of the spring network. Displacement of one of the end points would give an increase in energy. So the equilibrium state can be found by searching for the configuration of the springs where all derivatives with respect to one of the coordinates of an end point of a spring vanish. An exception has to be made for those end points which have some kind of constraint, for instance the presence of a wall. Only the 'free points' are free to move. The square lattice is the most simple two-dimensional lattice. The hamiltonian of an $N \times N$ lattice of springs with a vanishing eigenlength can be written as 


$$
H=\frac{C}{2} \sum_{i=0}^{N-1} \sum_{j=0}^{N-1}\left[\begin{array}{l}
\left(x_{i, j}-x_{i+1, j}\right)^{2}+\left(y_{i, j}-y_{i+1, j}\right)^{2}+ \\
\left.\left(x_{i, j}-x_{i, j+1}\right)^{2}+\left(y_{i, j}-y_{i, j+1}\right)^{2}\right]
\end{array}\right.
$$

Differentiation with respect to the free points, $j=0 . N-1$ and $j=1 . N-2$, of the hamiltonian

$$
\frac{d H}{d x_{i, j}}=0 \text { and } \frac{d H}{d y_{i, j}}=0
$$

gives a set of $2 N(N-2)$ linear equations

$$
\begin{aligned}
& 4 x_{i, j}-x_{i+1, j}-x_{i, j+1}-x_{i-1, j}-x_{i, j-1}=0 \\
& 4 y_{i, j}-y_{i+1, j}-y_{i, j+1}-y_{i-1, j}-y_{i, j-1}=0
\end{aligned}
$$

Together with the boundary conditions, for instance $y_{i, 0}=0,0 \leq i<N$, one can find the minimal energy configuration using linear algebra.

By introducing the eigenlength $U_{0}$ one obtains the hamiltonian

$$
\begin{array}{r}
H=\frac{C}{2} \sum_{i=0}^{N-1} \sum_{j=0}^{N-1}\left\{\left[\sqrt{\left(x_{i, j}-x_{i+1, j}\right)^{2}+\left(y_{i, j}-y_{i+1, j}\right)^{2}}-U_{0}\right]^{2}+\right. \\
\left.\left[\sqrt{\left(x_{i, j}-x_{i, j+1}\right)^{2}+\left(y_{i, j}-y_{i, j+1}\right)^{2}}-U_{0}\right]^{2}\right\}
\end{array}
$$

which gives a system of coupled non-linear equations if one uses (5). It is therefore impossible to apply the above simple linear algebra solution. After some preliminary studies we chose an algorithm that makes use of the forces of the individual springs in finding the minimal energy configuration. The method is described in more detail in the next paragraph. Since these calculations are rather timeconsuming we opted for a parallel implementation of the algorithm.

\section{Eigenlength model}

The program Trigrid simulates the behaviour of a porous amorphous material under pressure. It calculates the positions of the end points of Hooke-type springs with an eigenlength in a 2-D triangular network. The simulated particle has the form of a hexagon, which consists internally of a large number of congruent triangles. The springs are connected to each other on the triangle vertices. Initially, each spring has its eigenlength, which equals the triangle side. The porosity of the particle is modelled by the distribution of the springs across the triangles. The porous material modelled in this way is amorphous, i.e. non-crystalline. 
At a certain macroscopic strain the program Trigrid calculates iteratively the minimal energy configuration of the spring system. This is done in two phases. First the spring properties, i.e the stretch and orientation, are calculated from the coordinates of the end points of the springs. In the second phase all end points are shifted in an appropriate way. The iteration stops if the configuration approaches its equilibrium. The convergence criterion used is that the sum of the absolute displacements becomes small enough. After approaching an equilibrium, global information such as the force upon the network is calculated. If one or more springs have reached a length which is greater than the critical length, all those springs are broken and at the same macroscopic strain the configuration of the springs will be calculated again.

Before we go into deeper detail on the iterative finding of the minimal energy configuration we give a sketch of the main program.

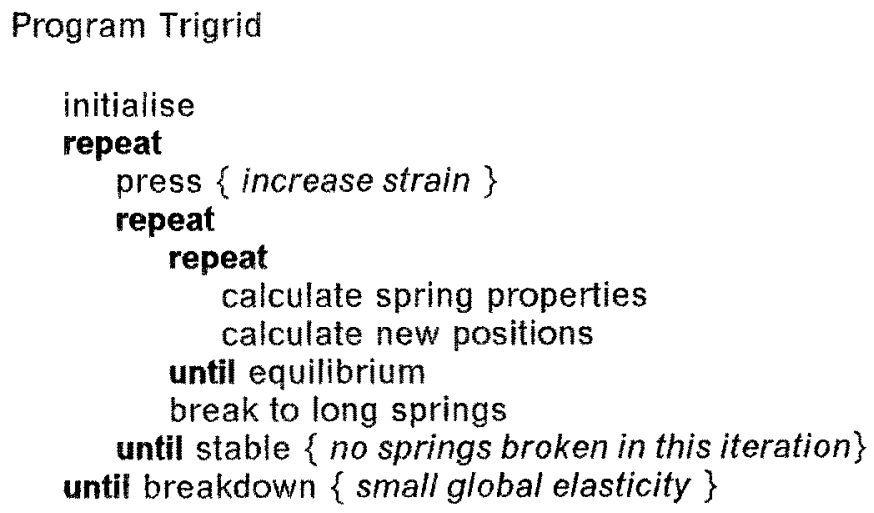

The status of every spring is represented by five fields: spring constant, eigenlength, stretch, $C_{x}$ eff and $C_{y}^{\text {eff }}$. Where $C_{x}^{\text {eff }}$ and $C_{y}^{\text {eff }}$ represent the orientation of the spring. $C_{x}^{e f f}$ is the inner product between a vector $C$ of length spring constant in the direction of the spring and a unit vector on the $x$-axis. After calculating the spring properties, the force on each point can be calculated as follows

$$
\begin{aligned}
& F_{x}=\sum_{\text {neighbours }} \text { stretch } \times c_{x}^{\text {eff }} \\
& F_{y}=\sum_{\text {neighbours }} \text { stretch } \times c_{y}^{\text {eff }}
\end{aligned}
$$

If two springs are attached to each other on one side and are bound at the other end point, there is a minimal energy configuration of this simple structure with the common end of the springs on the straight line between the two fixed end points. If the common end is displaced from the equilibrium position, the springs exert a force upon the common end. This force is proportional to the displacement from the equilibrium position. The corresponding effective spring constant depends on the 
direction of the displacement. Closer examination reveals that one needs to handle the movements in $x$ - and $y$-direction separately. The corresponding effective spring constants are

$$
\begin{aligned}
& c_{x}^{\text {eff }}=\sum_{\text {neighbours }}\left|c_{x}^{\text {eff }}\right| \\
& c_{y}^{\text {eff }}=\sum_{\text {neighbours }}\left|c_{y}^{\text {eff }}\right|
\end{aligned}
$$

Each point is shifted according to Hooke's Law

$$
\begin{aligned}
& \Delta x=F_{x} / C_{x}^{\text {eff }} \\
& \Delta y=F_{y} / C_{y}^{\text {eff }}
\end{aligned}
$$

\section{Parallel implementation}

In the parallel implementation, the initial hexagon is embedded in a parallelogram. This parallelogram is split up in $P \times P(P=6)$ smaller ones, and all computations for the springs contained in a single small parallelogram are performed by one process. Since springs from different small parallelograms can be connected; communications between processes dealing with the neighbouring parallelograms are needed. The small parallelograms have 2-D coordinates $(p . i, p . j)$, with $0 \leq p . i, p . j<P$. Two parallelograms $\left(p . i_{0}, p . j_{0}\right)$ and $\left(p . i_{1}, p . j_{1}\right)$ are neighbouring iff

$$
\left|p \cdot i_{0}-p \cdot i_{1}\right| \leq 1 \wedge\left|p \cdot j_{0}-p \cdot j_{1}\right| \leq 1
$$

Each process performs an iterative computation. At a certain macroscopic strain the configuration of the springs is calculated. First, the positions on the boundary of the parallelogram are communicated. The communications occur in two phases, one horizontal and one vertical, with the effect that the positions of the springs in parallelograms which touch only in one point $\left(\left|p . i_{0}-p . i_{1}\right|=1 \wedge\left|p . j_{0}-p . j_{1}\right|=1\right)$ are communicated over distance 2. This communication technique is well known; it is sometimes applied in domain decomposition computations [4].

Communication of spring properties never occurs; the only information that is communicated consists of the coordinates of the positions on the border. After the communications of the border positions, the spring properties, i.e the stretch and orientation, are calculated locally. All points are displaced according to (12) and (13). The sum of the absolute displacements is collected. The equilibrium is found if this sum is small enough.

After approaching an equilibrium, global information such as the force upon the network, is collected. If one or more springs have reached a length, which is 


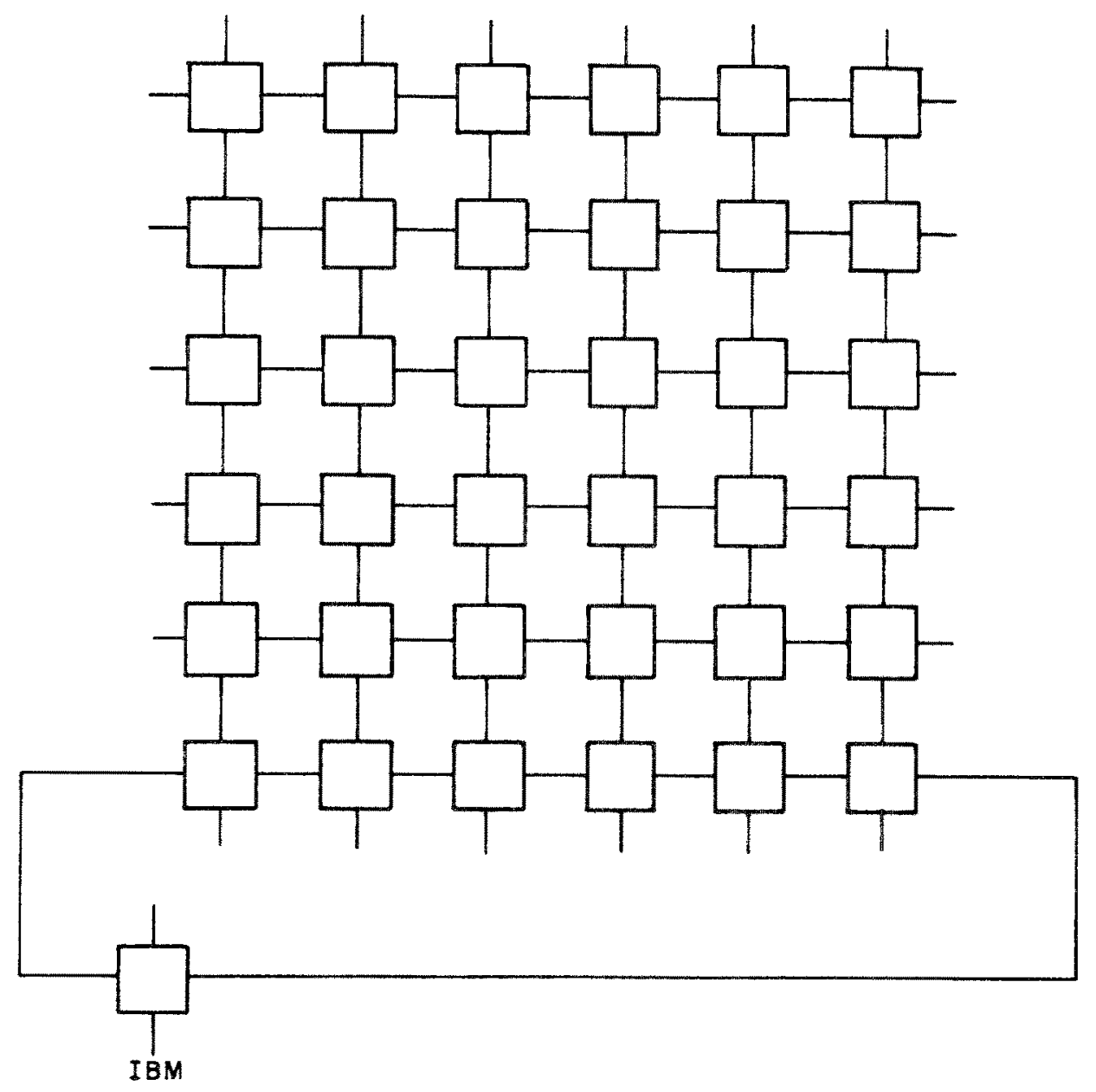

(Fig.2) The transputer system exists of 36 T800 transputers connected to each other in a mesh structure. Communication with the outside world is done via the root processor in a PC.

greater than the critical length, all those springs are broken and at the same macroscopic strain the configuration of the springs will be calculated again.

The distribution and collection of global information is done via the west border of the transputer network (Fig.2).

\section{Results}

The program Trigrid has been developed to perform calculations on a parallel transputer system ( 36 floating-point processors). Preliminary results were extracted from test calculations on a single transputer. 
If one starts with a non-porous structure, the structure is not amorphous but has a lot of symmetries. In consequence, the structure remains symmetric during the breaking process. We observed that the breaking process starts in the middle of the structure and propagates towards the border of the structure. For structures made of springs with a critical length that exceeds the eigenlength by 1 to 10 percent, the whole breaking process takes place at one strain. During the breaking process deformations in the spring structure occur. This can be made visible by plotting the broken and unbroken structures across each other (see Fig.3).

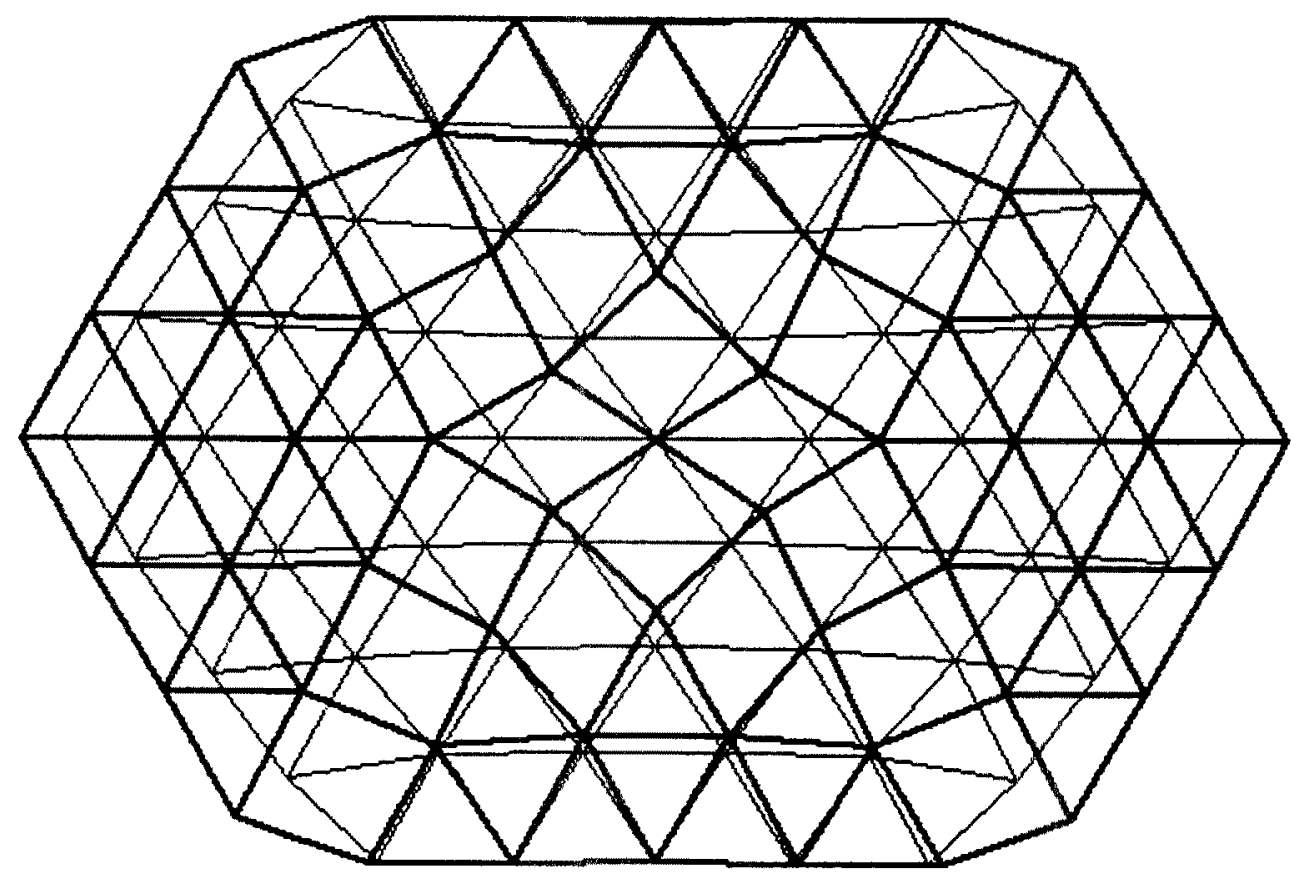

unbroken

broker

(Fig.3) A small system of 156 springs without porosity. Shown are the configurations of the springs just before and just after an avalanche of breakages. The critical length in this simulation is $10 \%$ above the eigenlength. Owing to this large critical length the deformation of the system is clearly visible.

The spring network and a porous amorphous material behave in a similar way. Increasing the strain leads to a higher stress intensity in the spring network. At a certain strain the length of one of the springs exceeds the critical length. The breaking of one spring may lead to a total collapse of the spring network at the same strain. In most cases global fallure is established after one or two avalanches of failures. For non-crystalline structures it is difficult to see if the system is broken or not. To make the failure better visible we chose another representation of the network. Instead of plotting lines for every unbroken spring we plot a coloured triangle if and only if at all three sides of the triangle an unbroken spring is present. A typical deformation experiment (Fig.4) has been performed on a network of 600 springs with an approximated porosity of $10 \%$. The breaking process starts in the 


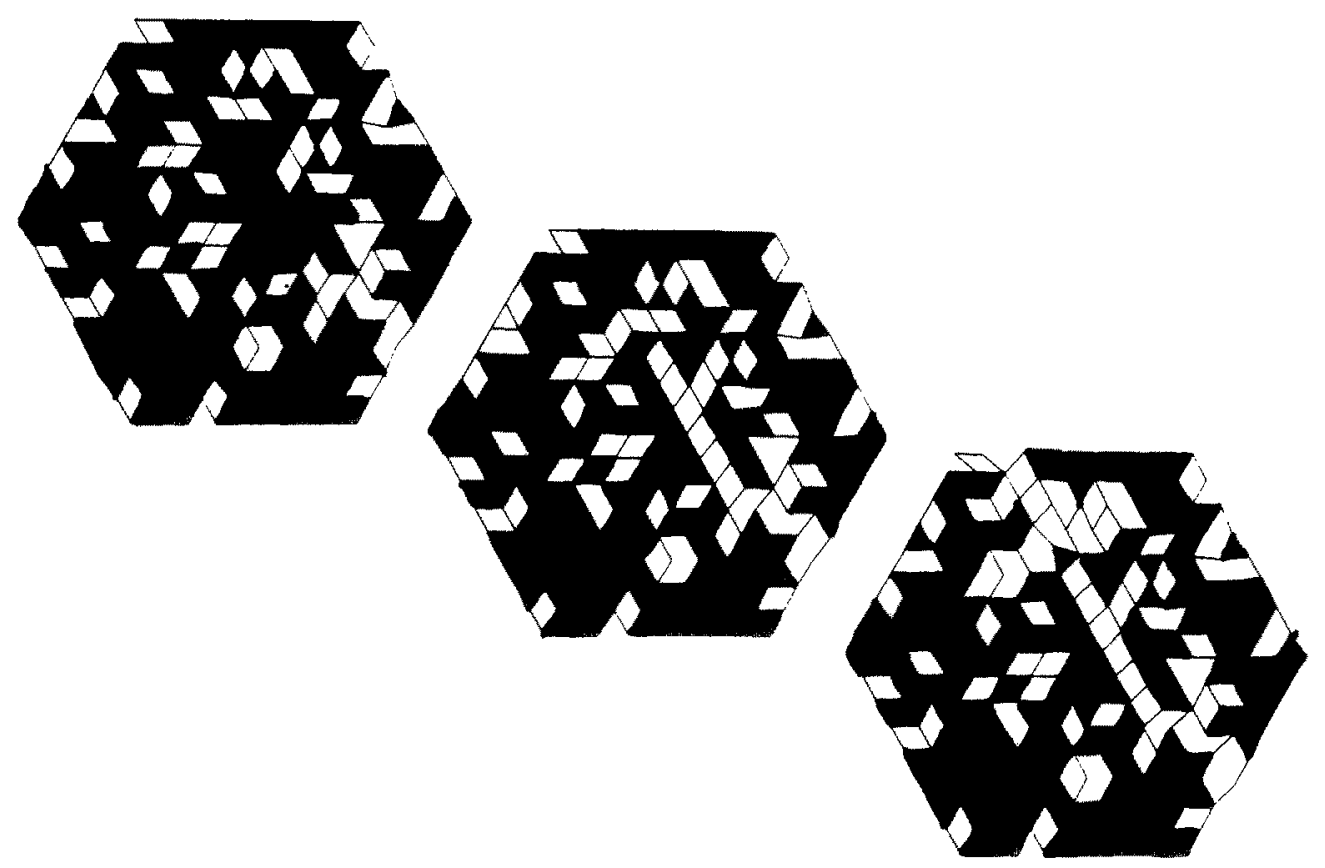

(Fig.4) A system of 600 springs with a porosity of $10 \%$. In the first configuration an avalanche of breakages is about to start. After two avalanches the system is almost broken. The critical length in this simulation is $1 \%$ above the eigenlength.

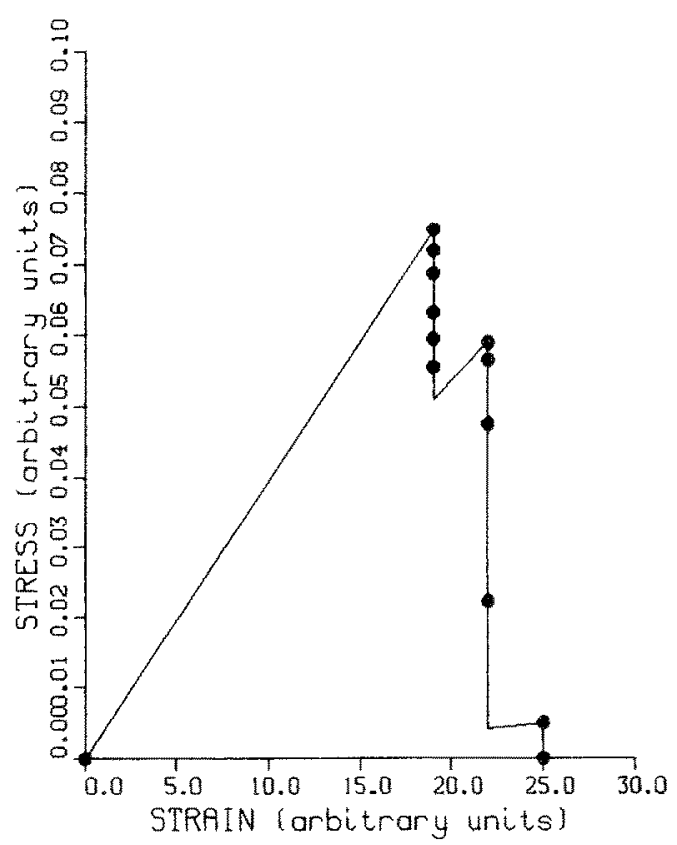

(Fig.5) The stress-strain behaviour of the same simulation as in Fig.4. 
middle of the structure and after two avalanches of failures the structure has fallen apart into two halves. The effective elasticity and strength can be obtained from the stress-strain behaviour of the same experiment (Fig.5).

Two results characterise the strength of the structure: $F_{\max }$, the maximum force on the structure and $d_{\max }$, the strain at the maximum force. In our model we can vary macroscopic parameters such as the form, size and porosity of the structure, as well as microscopic parameters such as the spring constant and critical length of an individual spring. Our goal is to develop a mathematical model that describes the impact of changing these parameters. This mathematical model can then be used for interpreting and guiding real deformation experiments on amorphous material.

As a start we look at the way in which the elasticity depends upon the porosity of the structure. In fact this is a well-known phenomenon and our results (Fig.6) do agree with results presented elsewhere. Since the elastic behaviour of the structure is a linear response behaviour, even analytical methods such as the effective medium approximation can be used to derive the porosity dependency of the elasticity. The elasticity of a structure decreases linearly towards the rigidity percolation threshold, i.e. at a porosity of about $35 \%[5]$.

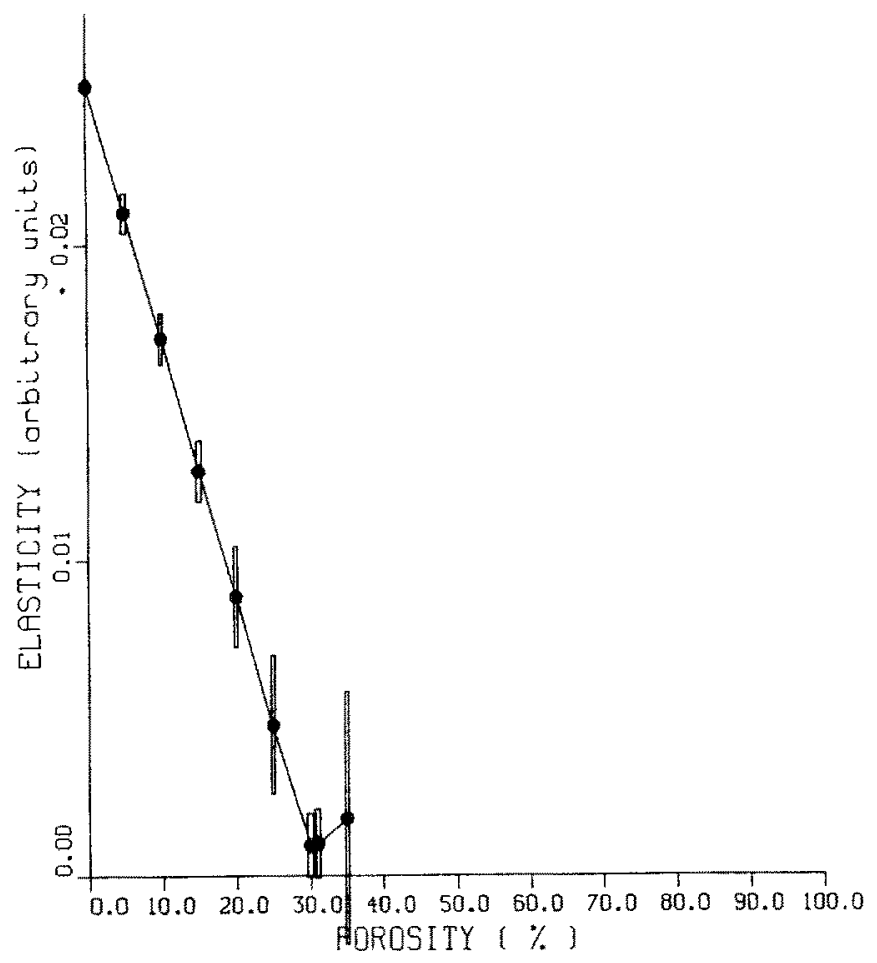

(Fig.6) Dependency of the elasticity on the porosity for a system of 600 springs of a hexagonal form in a triangular lattice.

More interesting are the results on the strength of the structure. Far more calculations are needed before we are able to understand these results. A spread in results is obtained no matter what representation is chosen. $A$ plot of $F_{\max }$ against 
$d_{\max }$ for different structures with the same porosity is seen as a cloud of points. The form and orientation of the cloud indicates that there is a correlation between these two variables. We should like to find two variables that are independent of each other. If two independent variables can be found, this may lead to a better understanding of the relative importance of the various parameters for the mechanical strength of the amorphous material. The distribution of these independent variables may be of a simple form. Preliminary results (Fig.7) indicate that $F_{\max } / d_{\max }$ and $\mathrm{d}_{\max }$ seem to be uncorrelated. These independent variables may be modelled separately and other variables of interest can be derived after taking the appropriate convolution.

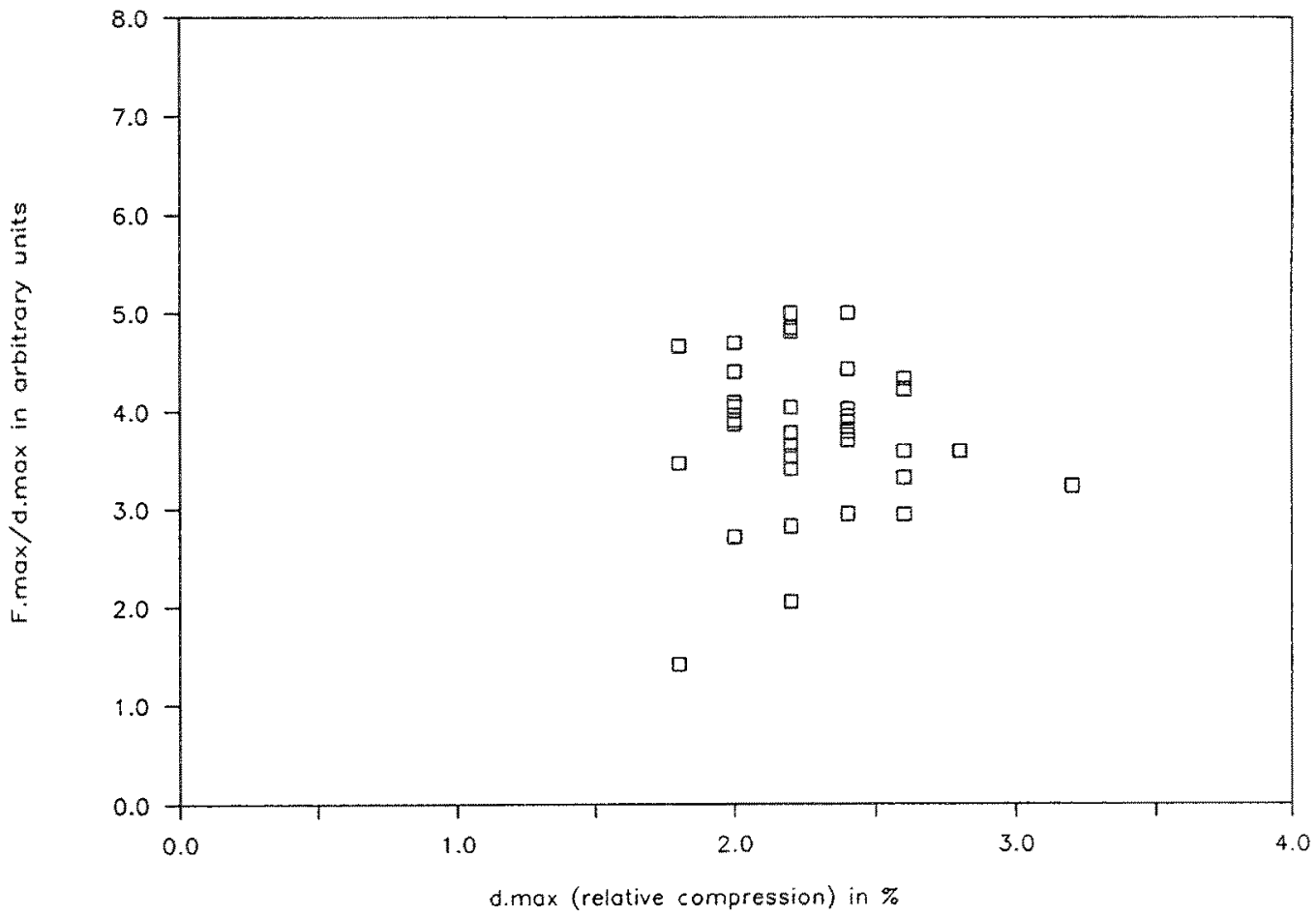

(Fig.7) Correlation between the maximum strength over the relative compression and the relative compression itself for a system of 600 springs with a porosity of $20 \%$. The critical length is $2 \%$ above the eigenlength.

Finally we conclude that the triangular spring model seems to be a good candidate to describe the mechanical strength of (porous) amorphous material. We aim to develop a mathematical model that describes the mechanical strength of amorphous material. The combination of computer simulations with real experiments seems to be worthwhile. A great many calculations are needed before we are able to obtain reliable statistics using finite size scaling methods. The only possibility is then to use cheap and fast computer facilities. Because of the short range interaction, parallelisation possibilities were embedded in the model. We established a linear speed up for our implementation, i.e. the same problem is solved $n$ times as fast on $n$ processors as on one processor only. 
After this paper had been presented at the Shell conference on parallel computing, we became aware of two new papers that have a close link to this work. Tang and Thorpe [6] examined the elastic properties of a random network of Hooke-type springs under tension. For small strains they determined the linear response behaviour in two ways: by simulation and by using an effective medium approximation. They were especially interested in the transition that takes place if the eigenlength is changed continuously from 0 to the initial spacing of the lattice. Beale and Srolovitz [7] analysed the distribution function of the breakdown stresses of a system of springs with vanishing eigenlength under tensile load. Their simulations indicate that the Duxbury-Leath distribution is more appropriate than the Weibull distribution. In contradiction to the less complicated tensile [7] and shear [3] loads, to our knowledge no simulations of failure mechanisms under compressive load have yet been published.

\section{Acknowledgements}

I am very grateful towards my colleagues C.E.D. Ouwerkerk and J.G.G. van de Vorst for the fruitful discussions and for their assistance on the experimental physics and the parallel computing side of the presented work, respectively.

\section{References}

[1] See e.g. M.A. Mull, A. Chudnovsky and A. Moet, "A probablistic approach to the fracture toughness of composites", Phil. Mag. A 56,419 (1987).

[2] D. Stauffer, "Introduction to Percolation Theory", Taylor and Francis, London, 1985.

[3] M. Sahimi and J.D. Goddard, "Elastic percolation models for cohesive mechanical failure in heterogeneous systems", Phys. Rev. B 33,7848 (1986).

[4] G.C. Fox, M.A. Johnson, G.A. Lyzenga, S.W. Otto, J.K. Salmon and D.W. Walker, "Solving Problems on Concurrent Processors", Vol. 1, Prentice-Hall, New Jersey, 1988.

[5] S. Feng, M.F. Thorpe and E. Garboczi, "Effective-medium theory of percolation on central-force elastic networks", Phys. Rev. B 31, 276 (1985).

[6] W. Tang and M.F. Thorpe, "Percolation of elastic networks under tension", Phys. Rev. B 37,5539 (1988).

[7] P.D. Beale and D.J. Srolovitz, "Elastic fracture in random materials", Phys. Rev. B 37, 5500 (1988). 